\title{
A REGULARITY RESULT FOR VISCOSITY SOLUTIONS OF HAMILTON-JACOBI EQUATIONS IN ONE SPACE DIMENSION
}

\author{
R. JENSEN AND P. E. SOUGANIDIS
}

\begin{abstract}
Viscosity solutions of Hamilton-Jacobi equations need only to be continuous. Here we prove that, in the special case of a one-dimensional stationary problem, under quite general assumptions, Lipschitz continuous viscosity solutions have right and left derivatives at every point. Moreover, these derivatives have some kind of continuity properties.
\end{abstract}

Introduction. The theory of nonlinear, first-order partial differential equations of Hamilton-Jacobi type has been substantially developed with the introduction by M. G. Crandall and P.-L. Lions [2] of the class of viscosity solutions, which turns out to be the correct class of generalized solutions for such equations. M. G. Crandall, L. C. Evans, and P.-L. Lions [1] provided a simpler introduction to the subject, while the book by P.-L. Lions [8] and the review paper by M. G. Crandall and P. E. Souganidis [3] provide a view of the scope of the theory and references to much of the recent literature.

Viscosity solutions need only to be continuous. On the other hand, they satisfy the corresponding equations at every point of differentiability. One would like to know, however, more about the structure of these solutions; for example, if and when there exist regions where viscosity solutions are (piecewise) continuously differentiable. Such kinds of results are of interest not only from the theoretical point of view but also for applications, since they may lead presumably to an understanding of the structure of the singularities of solutions of Hamilton-Jacobi type equations (e.g., the eikonal equation in geometrical optics, etc.).

This paper is concerned with the regularity of a Lipschitz continuous viscosity solution $u$ of the simple problem

$$
u+H\left(u_{x}\right)=v \quad \text { in }(-R, R)
$$

where $H: \mathbf{R} \rightarrow \mathbf{R}$ and $v:(-R, R) \rightarrow \mathbf{R}$ are continuous functions. The results obtained say that under some conditions on $H$ for every $x_{0} \in(-R, R) u$ has left and right derivatives at $x_{0}$, which we denote by $u_{x}^{-}\left(x_{0}\right)$ and $u_{x}^{+}\left(x_{0}\right)$ respectively. Moreover, these derivatives are continuous in a sense we make precise later.

Received by the editors April 4, 1986.

1980 Mathematics Subject Classification (1985 Revision). Primary 35L60, 35L99.

Key words and phrases. Hamilton-Jacobi equations, viscosity solutions, regularity.

The first author was partially supported by NSF under grant No. MCS-8001884A01; Alfred P. Sloan Fellow 1982.

The second author was partially supported by NSF under grant No. DMS-8401725 and ONR under contract No. N00014-83-K-0542. This work was completed while visiting the Institute for Mathematics and its Applications, University of Minnesota, Minneapolis, MN 55455. 
Before we state the results, we need to introduce some notation. In particular for $c \in \mathbf{R}$

$$
H^{-1}(\{c\})=\{p \in R: H(p)=c\}
$$

We have

THEOREM 1. Let $u$ be a locally Lipschitz continuous viscosity solution of (0.1), where $H: \mathbf{R} \rightarrow \mathbf{R}$ and $v:(R, R) \rightarrow \mathbf{R}$ are continuous functions. Assume that for every $c, M \in \mathbf{R}$ the set

$$
H^{-1}(\{c\}) \cap[-M, M]
$$

is either empty or finite and that the local extremum values (i.e., the local minimum and maximum values) of $H$ are isolated. Then for every $x_{0} \in(-R, R), u$ is either differentiable or it has left and right derivatives $u_{x}^{-}\left(x_{0}\right)$ and $u_{x}^{+}\left(x_{0}\right)$ at $x_{0}$ respectively. If $u_{x}^{-}\left(x_{0}\right) \neq u_{x}^{+}\left(x_{0}\right)$, then

$$
\left\{\begin{array}{l}
H\left(u_{x}^{-}\left(x_{0}\right)\right)=H\left(u_{x}^{+}\left(x_{0}\right)\right)=v\left(x_{0}\right)-u\left(x_{0}\right) \text { and } \\
H(l)\left(u_{x}^{+}\left(x_{0}\right)-u_{x}^{-}\left(x_{0}\right)\right) \geqslant\left(v\left(x_{0}\right)-u\left(x_{0}\right)\right)\left(u_{x}^{+}\left(x_{0}\right)-u_{x}^{-}\left(x_{0}\right)\right) \\
\quad \text { for every } l \in\left[\min \left(u_{x}^{-}\left(x_{0}\right), u_{x}^{+}\left(x_{0}\right)\right), \max \left(u_{x}^{-}\left(x_{0}\right), u_{x}^{+}\left(x_{0}\right)\right)\right] .
\end{array}\right.
$$

REMARK. The second part of (0.2) above is immediate from the definition of the viscosity solution.

THEOREM 2. Under the assumption of Theorem 1 for every $x_{0} \in(-R, R)$ there exists an $\alpha=\alpha\left(x_{0}\right)>0$ such that:

(i) If $v\left(x_{0}\right)-u\left(x_{0}\right)$ is not a local extremum value of $H$, then

$$
\left.u\right|_{\left(x_{0}-\alpha, x_{0}\right]} \in C^{1}\left(\left(x_{0}-\alpha, x_{0}\right]\right)^{1,2} \text { and }\left.u\right|_{\left[x_{0}, x_{0}+\alpha\right)} \in C^{1}\left(\left[x_{0}, x_{0}+\alpha\right)\right) \text {. }
$$

(ii) If $v\left(x_{0}\right)-u\left(x_{0}\right)$ is a local extremum value of $H$, then the following are true on $\left(x_{0}-\alpha, x_{0}\right]$ (respectively $\left[x_{0}, x_{0}+\alpha\right)$ ). Either $u(x)=v(x)+u\left(x_{0}\right)-v\left(x_{0}\right)$ or $u \mid\left(x_{0}-\alpha, x_{0}\right] \in C^{1}\left(\left(x_{0}-\alpha, x_{0}\right]\right)$ (respectively $\left.u\right|_{\left[x_{0}, x_{0}+\alpha\right)} \in C\left(\left[x_{0}, x_{0}+\alpha\right)\right)$ ) or $u_{x}(x)$ exists for every $x \in\left(x_{0}-\alpha, x_{0}\right]$ (respectively $\left[x_{0}, x_{0}+\alpha\right)$ ) except perhaps $a$ sequence $x_{n} \rightarrow x_{0}$ where $u_{x}^{-}\left(x_{n}\right) \neq u_{x}^{+}\left(x_{n}\right)$. In this last case, however, we have

$$
\lim _{\substack{x \uparrow x_{0} \\ x \in\left(x_{0}-\alpha, x_{0}\right]}} p(x)=u_{x}^{-}\left(x_{0}\right) \quad\left(\text { respectively } \lim _{\substack{x \downarrow x_{0} \\ x \in\left[x_{0}, x_{0}+\alpha\right)}} p(x)=u^{+}\left(x_{0}\right)\right),
$$

where $p(x) \in\left\{u_{x}^{-}(x), u_{x}^{+}(x)\right\}$.

Part (ii) asserts that in the case where $v\left(x_{0}\right)-u\left(x_{0}\right)$ is a local extremum value of $H$ one cannot expect in general something as simple as part (i). In particular, it may happen that there exist points arbitrarily close to $x_{0}$ where $u$ is not differentiable; the left and right derivatives at these points satisfy, however, the continuity property stated above. On the other hand, there are cases (for example $v \equiv 0$ ) where we know

$\left.{ }^{1} f\right|_{I}$ is the restriction of the function $f$ on $I$.

${ }^{2} C^{1}(I)$ is the space of continuously differentiable functions defined on $I$. 
that, independently of whether $v\left(x_{0}\right)-u\left(x_{0}\right)$ is a local extremum of $H$ or not, $u$ is continuously differentiable in a suitable neighborhood of any point $x_{0}$ with possible discontinuity of the derivative at $x_{0}$.

Inequality (0.2) is the Oleinik $E$-condition [9] as it applies to the problem (0.1) (for more details see §3). Thus Theorem 1 shows that the condition introduced by M. G. Crandall and P.-L. Lions in [2] to uniquely characterize solutions of Hamilton-Jacobi equations implies in one dimension the necessary condition for uniqueness of piecewise smooth solutions of scalar conservation laws.

The proof of Theorem 1 is based on a "blow-up" argument. More precisely, for $x_{0} \in(-R, R)$ and $\delta>0$ sufficiently small, let $u^{\delta}, v_{\delta}:[-1,1] \rightarrow \mathbf{R}$ be defined by

$$
\left\{\begin{array}{l}
u^{\delta}(x)=\frac{u\left(x_{0}+\delta x\right)-u\left(x_{0}\right)}{\delta} \text { and } \\
v_{\delta}(x)=v\left(x_{0}+\delta x\right) .
\end{array}\right.
$$

It turns out that $u^{\delta}$ is a viscosity solution of

$$
\delta u^{\delta}+H\left(u_{x}^{\delta}\right)=v_{\delta}-u\left(x_{0}\right) \text { in }(-1,1)
$$

The existence of left and right derivatives of $u$ at $x_{0}$ follows from an analysis of the possible values of $u_{x}^{\delta}$. The regularity of $u$ depends on the properties of $H$ and the fact that we work in one space dimension. All these are made precise in $\S 2$. In $\S 1$ we prove a kind of intermediate value theorem concerning sub- and superdifferentials of continuous functions defined in one space dimension. This is then used in the same section to obtain information about $u^{\delta}$ as well as Lipschitz continuoús viscosity solutions $u^{\infty}$ of the problem

$$
H\left(u_{x}^{\infty}\right)=v\left(x_{0}\right)-u\left(x_{0}\right) \text { in }(-R, R) .
$$

$\S 3$ describes the analogy with conservation laws.

General results concerning regularity of the value function of control problems were obtained by W. H. Fleming [6]. Very precise and general information about the structure of viscosity solutions of the corresponding to $(0.1)$ evolution problem in $\mathbf{R} \times(0, \infty)$ can be obtained by "integrating" the results of C. M. Dafermos [5] concerning the structure of solutions of conservation laws. For some results concerning the regularity of viscosity solution of certain special equations in two space dimensions, we refer to R. Jensen [7]. Finally, P. Cannarsa and H. M. Soner [4] recently obtained some regularity results concerning the evolution problem with convex Hamiltonian.

We conclude the introduction with the definitions of sub- and superdifferentials and viscosity solutions. We have

Definition $1[1,2]$. Let $f$ be a function from $(a, b)$ into $\mathbf{R}$ and let $x_{0} \in(a, b)$. Then the superdifferential of $f$ at $x_{0}$ is the set, denoted by $D^{+} f\left(x_{0}\right)$, of $p_{0} \in \mathbf{R}$ such that

$$
f(x) \leqslant f\left(x_{0}\right)+p_{0}\left(x-x_{0}\right)+o\left(\left|x-x_{0}\right|\right)
$$


holds. Similarly the subdifferential of $f$ at $x_{0}$ is the set, denoted by $D^{-} f\left(x_{0}\right)$, of $p_{0} \in \mathbf{R}$ such that

$$
f(x) \geqslant f\left(x_{0}\right)+p_{0}\left(x-x_{0}\right)+o\left(\left|x-x_{0}\right|\right)
$$

holds.

Definition $2[\mathbf{1}, 2]$. A continuous function $u: I \rightarrow \mathbf{R}$, where $I$ is an open subset of $\mathbf{R}$, is a viscosity solution of

$$
F\left(x, u, u_{x}\right)=0 \text { in } I
$$

if

$$
F(x, u(x), p) \leqslant 0, \quad \forall x \in I, \forall p \in D^{+} u(x)
$$

$$
F(x, u(x), p) \geqslant 0, \quad \forall x \in I, \forall p \in D^{-} u(x) .
$$

In a similar way a continuous function $u: I \rightarrow \mathbf{R}$ is said to be a viscosity subsolution (resp. supersolution) of (0.8) if (0.9) (resp. (0.10)) holds.

1. We begin with a lemma which plays the role of an intermediate value theorem concerning sub- and superdifferentials of continuous functions. The result is onedimensional and resembles the properties of Dini derivatives. (In one space dimension there is an obvious relation between sub- and superdifferentials and Dini derivatives). We have

LEMMA 1.1. Let $f \in C(I),{ }^{3}$ where $I$ is an open interval and $a, b \in I$ with $a<b$. The following are true:

(i) If $p \in D^{+} f(a), q \in D^{+} f(b)$ and $p<q$, then, for every $c \in(p, q)$, there exists a $x_{0} \in(a, b)$ such that $c \in D^{-} f\left(x_{0}\right)$.

(ii) If $p \in D^{-} f(a), q \in D^{-} f(b)$ and $p>q$, then, for every $c \in(q, p)$, there exists a $x_{0} \in(a, b)$ such that $c \in D^{+} f\left(x_{0}\right)$.

Proof. (i) Let $c \in(p, q)$ and define $g: I \rightarrow \mathbf{R}$ by

$$
g(x)=f(x)-c x \text {. }
$$

It is immediate that $p-c \in D^{+} g(a), q-c \in D^{+} g(b)$, and $p-c<0<q-c$. Using Definition 1 for $\varepsilon>0$ sufficiently small we obtain

$$
g(x)<g(a) \text { on }(a, a+\varepsilon) \text { and } g(x)<g(b) \text { on }(b-\varepsilon, b) \text {. }
$$

This implies that $g$ achieves a local minimum at some $x_{0} \in(a, b)$; therefore $0 \in D g\left(x_{0}\right)$; hence $c \in D^{-} f\left(x_{0}\right)$

(ii) The proof is similar to the one of (i); therefore we omit it.

We continue with a discussion of the properties of $u$ and $u^{\delta}$. Let $x_{0} \in(-R, R)$. If $u:(-R, R) \rightarrow \mathbf{R}$ is locally Lipschitz continuous, then $u^{\delta}, v_{\delta}:[-1,1] \rightarrow \mathbf{R}$ given by $(0.3)$ are well defined for sufficiently small $\delta>0$. Moreover, there exists a constant $L=L\left(x_{0}\right)>0$ such that

$$
\left\{\begin{array}{l}
\text { (i) }\left|u^{\delta}(x)\right| \leqslant L \quad \text { for every } x \in[-1,1] \text {, and } \\
\text { (ii) } u^{\delta} \text { is Lipschitz continuous with Lipschitz constant } L \text {. }
\end{array}\right.
$$

\footnotetext{
${ }^{3} C(I)$ is the space of continuous functions defined in $I \subset \mathbf{R}$.
} 
If $u$ is a viscosity solution of (0.1), then Definitions 1 and 2 imply that $u^{\delta}$ is a viscosity solution of (0.4). Finally, (1.1)(ii) and the properties of the viscosity solution [2], yield

$$
\left\{\begin{array}{l}
u+H\left(u_{x}\right)=v \quad \text { a.e. in }(-R, R) \text { and } \\
\delta u^{\delta}+H\left(u_{x}^{\delta}\right)=v_{\delta}-u\left(x_{0}\right) \text { a.e. in }(-1,1)
\end{array}\right.
$$

The next lemma is concerned with the possible values of $u_{x}^{\delta}$. The main idea is that $u^{\delta}$ being a viscosity solution of (1.2) forces $u_{x}^{\delta}$ not to "oscillate" too much. This follows from Lemma 1.1 and Definition 2. More precisely, we have

Lemma 1.2. Let $x_{0} \in(-R, R)$. Assume that $H, v$, and L are as in (1.1) and the statement of Theorem 1. For sufficiently small $\delta>0$ there exists a positive integer $n=n(\delta)$ such that for $i=0,1, \ldots, n$, then there exist $x_{i} \in[-1,1], \quad p_{i} \in$ $H^{-1}\left(\left\{v\left(x_{0}\right)-u\left(x_{0}\right)\right\}\right) \cap[-L, L]$, and mutually disjoint relatively open neighborhoods $I_{p_{i}}$ of $p_{i}$, in $[-L, L]$ with the properties:

(i) $-1=x_{0}<x_{1}<\cdots<x_{n-1}<x_{n}=1$;

(ii) $p_{1}<p_{2}<\cdots<p_{n-1}<p_{n}$;

(iii) ( $\alpha) u_{x}^{\delta} \in I_{p_{i}}$ a.e. on $\left[x_{i-1}, x_{i}\right]$ for every $i=1, \ldots, n$, or $(\beta) u_{x}^{\delta} \in I_{p_{n+1-i}}$ a.e. on $\left[x_{i-1}, x_{i}\right]$ for every $i=1, \ldots, n$.

In the case that (iii)( $\alpha)$ (respectively (iii) $(\beta)$ ) holds, if, for some $i, H\left(p_{i}\right)$ is a local extremum value of $H$, then it has to be a local minimum (respectively maximum). Moreover, $H(p) \geqslant v\left(x_{0}\right)-u\left(x_{0}\right)$ (respectively $H(p) \leqslant v\left(x_{0}\right)-u\left(x_{0}\right)$ ) for every $p \in\left[p_{1}, p_{n}\right]$. Finally, if $n \geqslant 2$, then, for $i=2, \ldots, n-1, H\left(p_{i}\right)$ is a local extremum value of $H$.

Proof. In view of (1.1) and (1.2) we have

$$
\left|H\left(u_{x}^{\delta}(x)\right)-\left(v\left(x_{0}\right)-u\left(x_{0}\right)\right)\right| \leqslant \omega_{v, x_{0}}(\delta)+\delta L \quad \text { for a.e. } x \in[-1,1],
$$

where $\omega_{v, x_{0}}(\cdot)$ is the modulus of continuity of $v$ at $x_{0}$ and $L$ is given by (1.1). The assumptions on $H$ imply that the set

$$
A=H^{-1}\left(\left\{v\left(x_{0}\right)-u\left(x_{0}\right)\right\} \cap[-L, L]\right.
$$

contains finitely many points $q_{1}<\cdots<q_{m}$ for some positive integer $m$. Moreover, by choosing $\delta$ sufficiently small we may assume that the restriction of $H$ on $[-L, L]$ has no local extremum values in $\left[v\left(x_{0}\right)-u\left(x_{0}\right)-\theta, v\left(x_{0}\right)-u\left(x_{0}\right)+\theta\right]$ except perhaps $H\left(q_{i}\right)$, where

$$
\theta=\omega_{v, x_{0}}(\delta)+\delta L
$$

Finally, for each $i=1, \ldots, m$ there exist mutually disjoint relatively open $[-L, L]$ neighborhoods $I_{q_{i}}$ of $q_{i}$ such that

$$
u_{x}^{\delta} \in \bigcup_{i=1}^{m} I_{q_{i}} \text { for a.e. } x \in[-1,1]
$$


and

$$
\left\{H(p): p \in I_{q_{i}}\right\}=\left\{\begin{array}{r}
\left(v\left(x_{0}\right)-u\left(x_{0}\right)-\theta, v\left(x_{0}\right)-u\left(x_{0}\right)+\theta\right) \\
\text { if } H\left(q_{i}\right) \text { is not a local extremum, } \\
{\left[v\left(x_{0}\right)-u\left(x_{0}\right), v\left(x_{0}\right)-u\left(x_{0}\right)+\theta\right)} \\
\text { if } H\left(q_{i}\right) \text { is a local minimum, } \\
\left(v\left(x_{0}\right)-u\left(x_{0}\right)-\theta, v\left(x_{0}\right)-u\left(x_{0}\right)\right] \\
\text { if } H\left(q_{i}\right) \text { is a local maximum. }
\end{array}\right.
$$

Let $\left\{p_{1}<\cdots<p_{n}\right\} \subset A$ be such that for each $i=1, \ldots, n$ there exists an $x \in(-1,1)$ with $u_{x}^{\delta}(x) \in I_{p_{i}}$. If $n=1$, there is nothing to show. If $n \geqslant 2$, let $x$, $y \in(-1,1)$ be such that $u_{x}^{\delta}(x) \in I_{p_{1}}, u_{x}^{\delta}(y) \in I_{p_{n}}$. Next we assume that $x<y$. Lemma 1.1(i), Definition 2, and (0.4), (1.1) yield

$$
H(c) \geqslant v\left(x_{0}\right)-u\left(x_{0}\right)-\theta \quad \text { for every } c \in\left[u_{x}^{\delta}(x), u_{x}^{\delta}(y)\right] .
$$

This implies that $H$ does not attain a local minimum value on $\left[p_{1}, p_{n}\right]$ except perhaps on some element of $A$ and that $H(c) \geqslant v\left(x_{0}\right)-u\left(x_{0}\right)$ for every $c \in\left[p_{1}, p_{n}\right]$. If there exists $z \in(-1, x)$ such that $u_{x}^{\delta}(z) \in I_{p_{i}}$ for $i>1$, then Lemma 1.1(ii), Definition 2, and (0.4), (1.1) yield

$$
H(c) \geqslant v\left(x_{0}\right)-u\left(x_{0}\right)=\theta \quad \text { for every } c \in\left[u_{x}^{\delta}(x), u_{x}^{\delta}(z)\right] .
$$

Combining (1.4), (1.5), and the way $\theta$ was chosen, we see that $H$ must be constant on $\left[u_{x}^{\delta}(x), \min \left(u_{x}^{\delta}(y), u_{x}^{\delta}(z)\right)\right]$ which is a contradiction. This shows that there exists $x_{1} \in(-1,1)$ such that $u_{x}^{\delta}(a) \in I_{p_{1}}$ for a.e. $a \in\left[-1, x_{1}\right]$. If there exist $w \in(y, 1)$ such that $u_{x}^{\delta}(w) \in I_{p_{j}}$ for $j<n$, arguing as above we again obtain a contradiction. So there exists $x_{n-1} \in(-1,1)$ such that $u_{x}^{\delta}(a) \in I_{p_{n}}$ for a.e. $a \in\left[x_{n-1}, 1\right]$. Next we reason in a similar way on $\left[x_{1}, x_{n-1}\right]$ and thus we obtain statements (i), (ii), and (iii) $(\alpha)$. Finally, it is immediate that for any two $p_{i}, p_{j}$ with $i<j, H$ cannot attain a local minimum on $\left(p_{i}, p_{j}\right)$; therefore if $H\left(p_{k}\right)$ is a local extremum value for $i \leqslant k \leqslant j$, it has to be a local minimum.

Case (iii) $(\beta)$ follows in exactly the same way if $x>y$ above.

The next result refines the information given by Lemma 1.2. In particular, it shows that as the whole family $\delta \downarrow 0$ either $n=1$ with $p_{1}$ independent of $\delta$ or $n=2$ with $x_{1}=0$ and $p_{1}, p_{2}$ independent of $\delta$. For notational simplicity in what follows we denote all subsequences of the family $\delta \downarrow 0$ in the same way as the family. We have:

Lemma 1.3. Let $x_{0} \in(-R, R)$ and $H, v, L$, and $A$ be as in (1.3) and Lemma 1.2. Then there exist $p, q \in A$ (independent of $\delta$ ) such that along the whole family $\delta \downarrow 0$ either

$$
u_{x}^{\delta} \in I_{p} \quad \text { for a.e. } x \in[-1,1]
$$

or

$$
u_{x}^{\delta} \in I_{p} \quad \text { for a.e. } x \in[-1,0] \text { and } u_{x}^{\delta} \in I_{q} \quad \text { for a.e. } x \in[0,1] \text {, }
$$


where $I_{p}, I_{q}$ are the neighborhoods of $p, q$ respectively defined in the proof of Lemma 1.2. Moreover, if (1.7) holds, then there exists a constant $c>0$ such that

$$
\left\{\begin{array}{l}
\text { If } p<q \text { (respectively } p>q) \text {, then } H \text { does not assume any values } \\
\text { smaller }(\text { respectively larger }) \text { than } v\left(x_{0}\right)-u\left(x_{0}\right)-c \text { (respectively } \\
\left.v\left(x_{0}\right)-u\left(x_{0}\right)+c\right) \text {. If } v\left(x_{0}\right)-u\left(x_{0}\right) \text { is a local extremum value of } \\
H \text { in either } I_{p} \text { or } I_{q} \text {, it has to be a local minimum (respectively } \\
\text { maximum). }
\end{array}\right.
$$

Proof of Lemma 1.3. We assume that there exists a subsequence $\delta \downarrow 0$ such that (iii) $(\alpha)$ of Lemma 1.2 holds. (If such a subsequence does not exist, then (iii) $(\beta)$ of Lemma 1.2 holds along the whole family $\delta \downarrow 0$. In this case, we argue in a similar fashion.) The fact that $A$ has finitely many points implies that along a further subsequence $\delta \downarrow 0$ we have $n(\delta)=n$ and for $i=1, \ldots, n$, whère $n$ and $p_{i} \in A$ are independent of $\delta$.

If $n=1$, then $u_{x}^{\delta} \in I_{p}$ for a.e. $x \in[-1,1]$. This yields $u_{x} \in I_{p}$ for a.e. $x \in$ $\left[x_{0}-\delta, x_{0}+\delta\right]$ as $\delta \downarrow 0$. For any arbitrary $\hat{\delta}>0$ such that $\hat{\delta}<\delta_{0}$, where $\delta_{0}$ is the largest element of the above subsequence, we have

$$
\left[x_{0}-\hat{\delta}, x_{0}-\hat{\delta}\right] \subset\left[x_{0}-\delta_{0}, x_{0}-\delta_{0}\right] ;
$$

thus $u_{x}^{\hat{\delta}} \in I_{p}$ for a.e. $x \in[-1,1]$.

If $n \geqslant 2$ we first show that, as $\delta \downarrow 0, x_{1}=x_{1}(\delta) \geqslant 0$. Indeed if not, then along a subsequence $\delta \downarrow 0$ we must have $-1<x_{1}<0$. This implies that, as $\delta \downarrow 0, u_{x}^{\delta} \in I_{p_{1}}$ for a.e. $x \in\left[x_{0}-\delta, x_{0}+\delta x_{1}\right]$ and $u_{x}^{\delta} \in \bigcup_{i=2}^{n} I_{p_{i}}$ for a.e. $x \in\left[x_{0}+\delta x_{1}, x_{0}+\delta\right]$ or equivalently, $u_{x} \in I_{p_{1}}$ for a.e. $x \in\left[x_{0}-\delta, x_{0}+\delta x_{1}\right]$ and $u_{x}^{\delta} \in \bigcup_{i=2}^{n} I_{p_{i}}$ for a.e. $x \in\left[x_{0}+\delta_{x_{1}}, x_{0}+\delta\right]$, where we may assume without any loss of generality that the neighborhoods $I_{p_{i}}$ above are independent of $\delta$. Next observe that $\delta x_{1}(\delta) \rightarrow 0$. Passing, if necessary, to a further subsequence, we may assume that $\delta x_{1}(\delta)$ strictly increases to 0 as $\delta \downarrow 0$. Let $\delta_{1}<\delta_{2}$ be such that $\delta_{1} x_{1}\left(\delta_{1}\right)>\delta_{2} x_{1}\left(\delta_{2}\right)$. The above analysis implies that

$$
u_{x} \in I_{p_{1}} \cap \bigcup_{i=2}^{n} I_{p_{i}} \quad \text { for a.e. } x \in\left[x_{0}+\delta_{2} x_{1}\left(\delta_{2}\right), x_{0}+\delta_{1} x_{1}\left(\delta_{1}\right)\right],
$$

which is a contradiction.

A similar argument shows that along a further, if necessary, subsequence $\delta \downarrow 0$, $x_{n-1}(\delta) \leqslant 0$. But then we have $n=2$ and $x_{1}=0$. For the general $\delta>0$ we argue as in the case where $n=1$. Finally, the rest of the assertions follow from Lemma 1.2 and its proof, thus we omit the details.

We conclude this section by discussing the nature of Lipschitz continuous viscosity solutions of (0.5). The results are very much similar to the ones of Lemma 1.2. For the proof of Theorem 1, however, we only need a less precise qualitative assertion which we give in the following lemma. Since the proof is similar to and simpler than the one of Lemma 1.2 we omit it. We have

LeMMA 1.4. Let $u^{\infty}$ be a Lipschitz continuous viscosity solution (0.5). Assume that $H$ is as in the statement of Theorem 1 . Then $u^{\infty}$ is linear or piecewise linear with finitely many corners. 
2. We begin with the proof of Theorem 1. As usual we denote subsequences in the same way as sequences. We have:

Proof of Theorem 1. Relation (1.1) yields that the family $\left\{u^{\delta}: \delta>0\right\}$ is precompact in $[-1,1]$, that is, along subsequences $u^{\delta}$ converges uniformly to Lipschitz continuous functions $u^{\infty}:[-1,1] \rightarrow \mathbf{R}$ which are ([2]) viscosity solutions of (0.5). Such solutions are described by Lemma 1.4. Here we use Lemma 1.3 to obtain that $u^{\infty}$ is unique and either linear or piecewise linear with only one corner at $x=0$, hence the existence of $u_{x}^{ \pm}\left(x_{0}\right)$.

To this end, assume that $x_{0}$ is such that (1.6) holds for $\delta>0$. Consider any $u^{\infty}:[-1,1] \rightarrow \mathbf{R}$ which is a possible limit of $\left\{u^{\delta}: \delta>0\right\}$ and suppose that there exist $x_{1}, x_{2} \in[-1,1]$ and $l \in A \backslash\{p\}$ such that $x_{1}<x_{2}$ and $u^{\infty}(x)=l x$ on $\left[x_{1}, x_{2}\right]$. We have

$$
\left(u^{\delta}\left(x_{2}\right)-u^{\infty}\left(x_{2}\right)\right)-\left(u^{\delta}\left(x_{1}\right)-u^{\infty}\left(x_{1}\right)\right)=\int_{x_{1}}^{x_{2}}\left(u_{y}^{\delta}(y)-l\right) d y .
$$

The left-hand side above tends to zero along a subsequence but the right-hand side does not; thus $u^{\infty}(x)=p x$ on $[-1,1]$ and $u_{x}\left(x_{0}\right)=p$. In the case that (1.7) holds for $\delta>0$, a similar argument shows that

$$
u^{\infty}(x)= \begin{cases}p x & \text { for } x \in[-1,0] \\ q s & \text { for } x \in[0,1]\end{cases}
$$

and, thus, the existence of $u_{x}^{ \pm}\left(x_{0}\right)$. Finally, the first part of $(0.2)$ is immediate from the properties of $u^{\infty}$.

We continue with Theorem 2 . The main step in the proof is to reduce to the case where we can invert $H$. If $H_{\alpha}^{-1}$ is an appropriate branch of $H_{\alpha}^{-1}$ and $u_{x}=H_{\alpha}^{-1}(v-u)$ a.e. in a neighborhood of $x_{0}$, then the continuity of the right-hand side will imply the result. Unfortunately this is not always possible; thus we have to deal with several cases. We have

Proof of Theorem 2. We begin with the case where $v\left(x_{0}\right)-u\left(x_{0}\right)$ is not a local extremum value of $H$. If $u_{x}\left(x_{0}\right)=p$ exists, then, for $x$ sufficiently close to $x_{0}, H^{-1}$ is defined in a neighborhood of $u\left(x_{0}\right)-v\left(x_{0}\right)$ with range some open neighborhood $I_{p}$ of $p$ and

$$
u(x)=u\left(x_{0}\right)+\int_{x_{0}}^{x} H^{-1}(v(y)-u(y)) d y,
$$

which implies the result. If $u_{x}^{-}\left(x_{0}\right)=p \neq u_{x}^{+}\left(x_{0}\right)=q$, then we have two branches $H_{p}^{-1}$ and $H_{q}^{-1}$ of the inverse of $H$ defined in a neighborhood of $v\left(x_{0}\right)-u\left(x_{0}\right)$ with values in some open disjoint neighborhoods $I_{p}$ and $I_{q}$ of $p$ and $q$ respectively. Again we have

$$
u(x)=u\left(x_{0}\right)-\int_{x}^{x_{0}} H_{p}^{-1}(v(y)-u(y)) d y \quad \text { for } x \leqslant x_{0}
$$

and

$$
u(x)=u\left(x_{0}\right)+\int_{x_{0}}^{x} H_{q}^{-1}(v(y)-u(y)) d y \quad \text { for } x \geqslant x_{0},
$$

thus the result. 
If $u\left(x_{0}\right)-v\left(x_{0}\right)$ is a local extremum of $H$ the argument is more complicated. We have to examine the following cases:

Case 1. $u_{x}^{-}\left(x_{0}\right)=p<q=u_{x}^{+}\left(x_{0}\right)$. We first assume that $H$ attains a local extremum only at $q$, which by Lemma 1.3 has to be a minimum. Moreover, $H$ is invertible in a neighborhood of $v\left(x_{0}\right)-u\left(x_{0}\right)$ with values in some $I_{p}$. Finally, Lemma 1.3 also yields the existence of an $\alpha>0$ such that $u_{x}(x) \in I_{p}$ for a.e. $x \in\left(x_{0}-\alpha, x_{0}\right]$ and $u(x) \in I_{q}$ for a.e. $x \in\left[x_{0}, x_{0}+\alpha\right)$, where $I_{q}$ is an appropriate open neighborhood of $q$. For $x<x_{0}$ we argue as before. For $x>x_{0}$ we define

$$
I_{q, 1}=\left\{l \in I_{q}: l \leqslant q\right\} \quad \text { and } \quad I_{q, 2}=\left\{l \in I_{q}: l \geqslant q\right\} .
$$

If, for a.e. $x \in\left[x_{0}, x_{0}+\alpha\right), u_{x}(x)$ either belongs to $I_{q, 1}$ or $I_{q, 2}$, then we can again invert $H$ and argue as before. If this does not happen, then we cannot necessarily show that $u$ is of class $C^{1}$ for $x>x_{0}$ in general. On the other hand, we are able to obtain some other information concerning $q$. In particular, we can find $x_{0}<x<y$ $<x_{0}+\alpha$ arbitrarily close to $x_{0}$ such that $u_{x}(x) \in I_{q, 1} \backslash\{q\}$ and $u_{x}(y) \in$ $I_{q, 2} \backslash\{q\}$. Then Lemma 1.1 implies the existence of $z$ such that $x<z<y$ and $q \in D \bar{u}(z)$. This yields $u(z)+H(q) \geqslant v(z)$. But $u(x)+H\left(u_{x}\right)=v(x)$ and $H(q)$ $\leqslant H\left(u_{x}\right)$ for a.e. $x \in\left[x_{0}, x_{0}+\alpha\right)$. The continuity of $u$ and $v$ then imply

$$
u(x)-u\left(x_{0}\right) \leqslant v(x)-v\left(x_{0}\right) \text { for every } x \in\left[x_{0}, x_{0}+\alpha\right) .
$$

Combining all the above we obtain

$$
u(z)-u\left(x_{0}\right)=v(z)-v\left(x_{0}\right)
$$

and, moreover,

$$
q=\lim _{x \downarrow x_{0}} \frac{v(x)-v\left(x_{0}\right)}{x-x_{0}}
$$

Next define the sets

$$
\mathrm{I}=\left\{x \in\left[x_{0}, x_{0}+\alpha\right]: u(x)-u\left(x_{0}\right)=v(x)-v\left(x_{0}\right)\right\}
$$

and

$$
\mathrm{II}=\left\{x \in\left[x_{0}, x_{0}+\alpha\right]: u(x)-u\left(x_{0}\right)<v(x)-v\left(x_{0}\right)\right\} .
$$

We first investigate the behavior of $u$ on $\mathrm{I}$, which is closed and nonempty. Let $z \in \mathrm{I}$. By Theorem $1 u_{x}^{ \pm}(z)$ exists and lies in a small neighborhood of $q$ provided that $\alpha$ is sufficiently small. On the other hand, since $H\left(u_{x}^{ \pm}(z)\right)=v(z)-u(z)=v\left(x_{0}\right)-$ $u\left(x_{0}\right)$, the properties of $H$ yield $u_{x}^{ \pm}(z)=q$; thus $u$ is differentiable at $z$. If $\mathrm{I}=\left[x_{0}, x_{0}+\alpha\right]$ there is nothing to show. If not, then II is a nonempty open set; therefore it can be written as countable union of mutually disjoint open intervals $J_{m}$, i.e., $\mathrm{II}=\bigcup_{m} J_{m}$. On each $J_{m}=\left(a_{m}, b_{m}\right)$ we have $u(x)-u\left(x_{0}\right)<v(x)-v\left(x_{0}\right)$ and

$$
\begin{aligned}
u\left(a_{m}\right)-u\left(x_{0}\right) & =u\left(b_{m}\right)-u\left(x_{0}\right)=v\left(a_{m}\right)-v\left(x_{0}\right) \\
& =v\left(b_{m}\right)-v\left(x_{0}\right)=u\left(x_{0}\right)-v\left(x_{0}\right) .
\end{aligned}
$$


It is easy to see arguing as before that for every $x \in J_{m}$ either $u_{x}^{ \pm}(x) \in I_{q, 1} \backslash\{q\}$ or $u_{x}^{ \pm}(x) \in I_{q, 2} \backslash\{q\}$. Moreover, Lemma 1.1 yields that there exists at most one $c_{m} \in J_{m}$ such that

$$
u_{x}(x) \in I_{q, 2} \backslash\{q\} \text { for a.e. } x \in\left(a_{m}, c_{m}\right)
$$

and

$$
u_{x}(x) \in I_{q, 1} \backslash\{q\} \quad \text { for a.e. } x \in\left(c_{m}, b_{m}\right) .
$$

Arguments similar to the ones at the beginning of the proof imply that

$$
u \in C^{1}\left(\left(a_{m}, c_{m}\right)\right) \text { and } u \in C^{1}\left(\left(c_{m}, b_{m}\right)\right) .
$$

Moreover, $u_{x}^{-}\left(c_{m}\right) \neq u_{x}^{+}\left(c_{m}\right)$ and $\lim _{x \rightarrow a_{m}} u_{x}(x)=\lim _{x \rightarrow b_{m}} u_{x}(x)=q$. This completes the proof.

If $p$ is a local minimum but $q$ is not or when both are local minima, we argue as above changing (2.1) appropriately.

The remaining two cases,

Case 2. $u_{x}^{-}\left(x_{0}\right)=p>u_{x}^{+}\left(x_{0}\right)=q$ and

Case 3. $u_{x}\left(x_{0}\right)=p$,

follow in the same way; therefore we omit the details.

We conclude this section by showing how one can overcome the above difficulties in the special case where $v \equiv 0$. We have

Proposition 2.1. Under the assumption of Theorem 2 but with $v \equiv 0$, for every $x_{0} \in(-R, R)$ there exists an $\alpha=\alpha\left(x_{0}\right)>0$ such that

$$
\left.u\right|_{\left(x_{0}-\alpha, x_{0}\right]} \in C^{1}\left(\left(x_{0}-\alpha, x_{0}\right]\right) \text { and }\left.u\right|_{\left[x_{0}, x_{0}+\alpha\right)} \in C^{1}\left(\left[x_{0}, x_{0}+\alpha\right)\right) \text {. }
$$

Proof. We continue with the proof of Case 1 of Theorem 2. We want to claim that $u \equiv u\left(x_{0}\right)$ on $\left[x_{0}, z\right]$. Indeed if not then there exists a $w \in\left(x_{0}, z\right)$ such that $u(w)<u\left(x_{0}\right)=u(z)$ and $0 \in D^{-} u(w)$. But then $u(w)+H(0) \geqslant 0$. On the other hand, by (2.1), $q=0$ and $H(q)=-u\left(x_{0}\right)$. Therefore $u(w) \geqslant u\left(x_{0}\right)$, which leads to a contradiction, thus the result. The other cases can be treated similarly.

3. In this section we use Theorem 1 to illustrate the relation between the notion of viscosity solution and the $E$ condition introduced in [9] by O. A. Oleinik. Throughout the section we assume

$$
\left\{\begin{array}{l}
\text { (i) } H, v \text { are continuously differentiable; } \\
\text { (ii) } u\left(x_{0}\right)-v\left(x_{0}\right) \text { is not a local extremum value of } H .
\end{array}\right.
$$

Let $w:(-R, R) \rightarrow \mathbf{R}$ be defined by

$$
w(x)=u_{x}(x) \text { for a.e. } x \in(-R, R) .
$$

In view of (3.1) and the results of the previous section, at every point where $u_{x}$ exists, $w$ is continuous and it satisfies

$$
w+(H(w)-v)_{x}=0 \text { a.e. in }(-R, R) .
$$


Moreover, at points where $u_{x}$ does not exist, $w$ has right and left limits $w_{+}$and $w_{-}$ respectively which satisfy

$$
\left\{\begin{array}{l}
H\left(w_{+}\right)=H\left(w_{-}\right)=v\left(x_{0}\right)-u\left(x_{0}\right) \text { and } \\
H(p) \leqslant H\left(w_{+}\right) \text {for every } p \in\left[w_{+}, w_{-}\right] \text {if } w_{-}>w_{+} \text {or } \\
H(p) \geqslant H\left(w_{-}\right) \text {for every } p \in\left[w_{-}, w_{+}\right] \text {if } w_{+}>w_{-}
\end{array}\right.
$$

Relation (3.3), however, is the E condition, as it applies to (3.2). Indeed let

$$
l(w)=\frac{\left(H\left(w_{+}\right)-v\left(x_{0}\right)\right)-\left(H\left(w_{-}\right)-v\left(x_{0}\right)\right)}{w_{+}-w_{-}}\left(w-w_{+}\right)+H\left(u_{+}\right)-v\left(x_{0}\right) .
$$

We say (cf. O. A. Oleinik [9]) that a solution $w$ of (3.2) satisfies the $E$ condition if at all points of discontinuity $x_{0}$ of $w$, the following is satisfied: If $w_{+}>w_{-}$, then $l(w) \leqslant H(w)-v\left(x_{0}\right)$ for all $w \in\left[w_{-}, w_{+}\right]$and if $w_{-}>w_{+}$, then $l(w) \geqslant H(w)-$ $v\left(x_{0}\right)$ for all $w \in\left[w_{+}, w_{-}\right]$.

\section{REFERENCES}

1. M. G. Crandall, L. C. Evans, and P.-L. Lions, Some properties of viscosity solutions of Hamilton-Jacobi equations, Trans. Amer. Math. Soc. 282 (1984), 487-502.

2. M. G. Crandall and P.-L. Lions, Viscosity solutions of Hamilton-Jacobi equations, Trans. Amer. Math. Soc. 277 (1983), 1-42.

3. M. G. Crandall and P. E. Souganidis, Developments in the theory of nonlinear first-order partial differential equations, Proc. Internat. Sympos. on Differential Equations (Birmingham, Alabama, 1983), I. W. Knowles and R. T. Lewis, Eds., North-Holland, Amsterdam, 1984.

4. P. Cannarsa and H. M. Soner. On the singularities of the viscosity solutions to Hamilton-Jacobi-Bellman equations, IMA Preprint Series \#201 (to appear).

5. C. M. Dafermos, Regularity and large time behaviour of solutions of a conservation law without convexity (to appear).

6. W. H. Fleming, The Cauchy problem for a nonlinear first-order partial differential equation, J. Differential Equations 5 (1969), 515-530.

7. R. Jensen, in preparation.

8. P.-L. Lions, Generalized solutions of Hamilton-Jacobi equations, Pitman, Boston, Mass., 1982.

9. O. A. Oleinik, Uniqueness and stability of the generalized solution of the Cauchy problem for a quasilinear equation, Amer. Math. Soc. Transl. (2) 33 (1963), 285-290.

Department of Mathematics, University of Kentucky, LeXington, Kentucky 40506

Lefschetz Center for Dynamical Systems, Division of Applied Mathematics, Brown UniverSITY, PROVIDENCE, RHODE ISLAND 02912 